Santos WS, Gonçalves NJMC \& Viana MRP (2020). Nursing assistance to patients with acute myocardial infarction submitted to coronary angioplasty. Research, Society and Development, 9(7): 1-13, e755974878.

\title{
Assistência de Enfermagem a paciente com infarto agudo do miocárdio submetido a angioplastia coronariana
}

\section{Nursing assistance to patients with acute myocardial infarction submitted to coronary angioplasty \\ Asistencia de Enfermería a pacientes con infarto miocardio agudo presentado a angioplastia coronaria}

Recebido: 24/05/2020 | Revisado: 27/05/2020 | Aceito: 27/05/2020 | Publicado: 11/06/2020

William da Silva Santos

ORCID: https://orcid.org/0000-0002-0135-0286

Centro Universitário Santo Agostinho, Brasil

E-mail: mano-campelo77@ @otmail.com

Nyckson Josué de Meneses Claudino Gonçalves

ORCID: https://orcid.org/0000-0001-6821-6842

Centro Universitário Santo Agostinho, Brasil

E-mail: nycksonjosue99@gmail.com

Magda Rogéria Pereira Viana

ORCID: https://orcid.org/0000-0003-3293-7095

Centro Universitário Santo Agostinho, Brasil

E-mail: magdarogeria@hotmail.com

\section{Resumo}

Descrever a assistência de enfermagem ao paciente com infarto agudo do miocárdio submetido à angioplastia coronariana evidenciada nas publicações científicas e analisar como é realizada a assistência de enfermagem prestada ao paciente com infarto agudo do miocárdio submetido à angioplastia coronariana de acordo com as publicações científicas. Trata-se de um estudo de revisão integrativa da literatura, com abordagem qualitativa. A coleta de dados foi realizada no mês de março de 2020 nos bancos de dados da Biblioteca Virtual em Saúde (BVS), nas bases de dados SCIELO, LILACS e Google acadêmico, com os seguintes descritores presentes no DECS: Assistência de Enfermagem, Infarto Agudo do Miocárdio, Angioplastia. Como critérios de inclusão foram escolhidos os artigos que apresentavam texto 
completo, em português e espanhol, no período de 2014 a 2019 e que estavam relacionados ao tema. Foram excluídos: teses, dissertações, artigos pagos, de jornal e textos que não apresentavam relação com a temática e revisões de literatura. Ao final da amostra foram obtidos 9 artigos, que foram analisados criteriosamente. O estudo foi dividido em duas categorias em que a primeira mostra o Perfil de pacientes com Infarto Agudo do Miocárdio e as Implicações para assistência de Enfermagem e a segunda aborda sobre a assistência de Enfermagem ao paciente submetido a angioplastia coronariana pós Infarto Agudo do Miocárdio. Diante do exposto estudo atendeu aos objetivos propostos e mostrou-se de grande relevância, pois poderá contribuir para o entendimento do enfermeiro na abordagem e na atenção ao paciente com IAM submetido à angioplastia coronariana.

Palavras-chave: Assistência de Enfermagem; Infarto agudo do miocárdio; Angioplastia.

\begin{abstract}
Describe nursing care for patients with acute myocardial infarction undergoing coronary angioplasty as evidenced in scientific publications and analyze how nursing care provided to patients with acute myocardial infarction undergoing coronary angioplasty is carried out according to scientific publications. It is an integrative literature review study, with a qualitative approach. Data collection was carried out in March two thousand twenty in the databases of the Virtual Health Library (VHL), in the SCIELO, LILACS and Google academic databases, with the following descriptors present in the DECS: Nursing Care, Acute Infarction of the Myocardium, Angioplasty. As inclusion criteria, articles with full text, in Portuguese and Spanish, from two thousand fourteen to two thousand nineteen and related to the theme were chosen. The following were excluded: theses, dissertations, paid articles, newspaper articles and texts that were not related to the theme and literature reviews. At the end of the sample, nine articles were obtained, which were carefully analyzed. The study was divided into two categories in which the first shows the Profile of patients with Acute Myocardial Infarction and the Implications for Nursing care and the second deals with Nursing care for patients undergoing coronary angioplasty after Acute Myocardial Infarction. In view of the above study, it met the proposed objectives and proved to be of great relevance, as it may contribute to the understanding of nurses in the approach and care for patients with
\end{abstract} AMI undergoing coronary angioplasty.

Keywords: Nursing care; Acute myocardial infarction; Angioplasty. 


\section{Resumen}

Describa la atención de enfermería para pacientes con infarto agudo de miocardio que se someten a angioplastia coronaria como se evidencia en publicaciones científicas y analice cómo la atención de enfermería brindada a pacientes con infarto agudo de miocardio que se somete a angioplastia coronaria se lleva a cabo de acuerdo con publicaciones científicas. Es un estudio integrador de revisión de literatura, con un enfoque cualitativo. La recolección de datos se realizó en marzo de 2020 en las bases de datos de la Biblioteca Virtual en Salud (BVS), en las bases de datos académicas SCIELO, LILACS y Google, con los siguientes descriptores presentes en el DECS: Atención de enfermería, infarto agudo del miocardio, angioplastia. Como criterios de inclusión, se eligieron artículos con texto completo, en portugués y español, de 2014 a 2019 y relacionados con el tema. Se excluyeron los siguientes: tesis, disertaciones, artículos pagados, artículos de periódicos y textos que no estaban relacionados con el tema y las revisiones de literatura. Al final de la muestra, se obtuvieron 9 artículos, que fueron analizados cuidadosamente. El estudio se dividió en dos categorías en las que la primera muestra el Perfil de pacientes con infarto agudo de miocardio y las implicaciones para la atención de enfermería y la segunda trata con la atención de enfermería para pacientes sometidos a angioplastia coronaria después de infarto agudo de miocardio. Dado el estudio anterior, cumplió con los objetivos propuestos y demostró ser de gran relevancia, ya que puede contribuir a la comprensión de las enfermeras en el enfoque y la atención de pacientes con IAM sometidos a angioplastia coronaria.

Palabras clave: Atención de Enfermería; Infarto agudo de miocardio; Angioplastia.

\section{Introdução}

As doenças cardiovasculares (DCV) são consideradas um grave problema que acomete a população em nível global, tornando-se a principal causa de morbimortalidade em países desenvolvidos e em desenvolvimento. O conjunto das doenças cardiovasculares representa hoje a maior causa de perdas de anos de vida em todo o mundo, bem como no Brasil, pois muitas pessoas ainda adoecem e morrem por agravamentos dessas patologias, prevalecendo como um problema de saúde pública no país (Brasil, 2017).

De acordo com Barroso et al., (2017), dentre as DCV, a Hipertensão Arterial Sistêmica (HAS) constitui importante fator de risco para complicações cardíacas, que atinge cerca de 32,5\% da população adulta no Brasil. Outra patologia a ser citada é Diabetes Mellitus, que em 2014 contabilizou dados com cerca de 11,9 milhões de brasileiros 
acometidos pela doença. $\mathrm{O}$ uso do fumo é apontado como fator em $25 \%$ de toda a população adulta mundial tendo boa participação na epidemiologia das DCV. Dados da OMS (2015) apontam que 17,7 milhões de pessoas morreram por doenças cardiovasculares em nível global. Desses óbitos, estima-se que 7,4 milhões ocorrem devido às doenças coronarianas. No Brasil, as DCV são responsáveis por $29,4 \%$ de todas as mortes registradas por ano (Lima et al., 2018).

As DCV são um grupo de doenças que acometem o funcionamento do coração e vasos sanguíneos que irrigam o músculo cardíaco, membros superiores e inferiores, que são ocasionados por depósitos de gorduras no lúmen dos vasos promovendo a obstrução da circulação efetiva, condição essa que caracteriza a aterosclerose, um quadro patológico que com o passar do tempo ocasiona uma isquemia no músculo cardíaco, iniciando assim o infarto agudo do miocárdio situação grave, que necessita de assistência imediata (Brasil, 2017).

Em pacientes com agravamento do Infarto Agudo do Miocárdio (IAM), com supra desnivelamento do segmento ST, pode ser realizado o tratamento não cirúrgico, a angioplastia coronariana, que é feita através de um cateter com um pequeno balão, puncionado ou na artéria radial ou femoral introduzido nas coronárias, seguindo até a obstrução onde infla e desinfla algumas vezes, comprimindo a placa e melhorando a repercussão sanguínea (Mendes \& Miranda, 2015).

A Angioplastia Coronariana foi realizada pela primeira vez por Gruntzig em 1977, utilizando cateteres de duplo lúmen, dotados de um balão na ponta distal, comprimindo a placa de ateroma contra a parede da artéria. Esta desobstrução é conseguida pela insuflação do balão, sob alta pressão, ao nível do ateroma. No Brasil, foi realizada pela primeira vez em 1979, em Curitiba, por Costantini e colaboradores, em um paciente de 55 anos. Após isso, a Angioplastia Coronariana ganhou ampla aceitação em todo o mundo, particularmente no ocidental, onde são executados cerca de 400.000 procedimentos ao ano (Taets, 2016)

O tratamento com Angioplastia Coronariana tem sido utilizado como conservador em pacientes com DCV, mostrando-se ser um método eficaz e seguro de revascularização do miocárdio, promovendo resultados significantes na melhora do quadro clinico de pacientes com lesões coronarianas complexas. Tem expressado uma melhora na qualidade de vida relacionada à diminuição dos sintomas, oferece maior conforto ao paciente, melhorando sua mobilização, deambulação precoce e menor custo hospitalar (Régis, Rosa, \& Lunelli, 2017).

Dessa forma, torna-se importante uma assistência de Enfermagem de qualidade ao paciente no pós-Angioplastia Coronariana, com intervenções especificas, caso o paciente 
apresente complicações sistêmicas, identificando e minimizando seus efeitos, reduzindo desconfortos e contribuindo para uma assistência holística ao paciente (Mendes \& Miranda, 2015).

A atuação da Enfermagem nos cuidados a pacientes submetidos à Angioplastia Coronariana torna-se imprescindível, pois o Enfermeiro tem sua atuação em geral como gerente da equipe, tomando as devidas providencias para a correta realização do procedimento. Além disso, atua nos cuidados assistenciais a partir da consulta de Enfermagem, promovendo orientações ao paciente esclarecendo possíveis dúvidas, minimizando fatores de riscos associados à ocorrência de complicações pós-realização, como é o caso de alergias ao contraste iodado além da exposição ionizante (Kuhn et al., 2015)

Com isso, é necessário destacar a relevância do estudo, por sua escassez e pelo respaldo que irá proporcionar a outros pesquisadores em buscar novas atualizações, para satisfazer suas curiosidades em relação a um tema que representa grande significado para o desenvolvimento de atitudes tomadas pelos Enfermeiros em pacientes submetidos à angioplastia coronariana.

Diante do exposto, o estudo tem como objeto a assistência de enfermagem ao paciente com infarto agudo do miocárdio submetido à angioplastia coronariana. Como questão para revisão: qual a assistência de enfermagem ao paciente com infarto agudo do miocárdio submetido à angioplastia coronariana? Para responder a esta questão elencaramse os objetivos: descrever a assistência de enfermagem ao paciente com infarto agudo do miocárdio submetido à angioplastia coronariana evidenciada nas publicações científicas e analisar como é realizada a assistência de enfermagem prestada ao paciente com infarto agudo do miocárdio submetido à angioplastia coronariana de acordo com as publicações científicas.

\section{Metodologia}

Trata-se de um estudo de revisão de literatura, pelo método de revisão integrativa com abordagem qualitativa. De acordo com Galvão \& Pereira (2014), tem sido utilizada como recurso metodológico, a qual faz uso de estratégia sistematizada para agrupar e produzir resultados de estudos sobre um determinado tema, com o objetivo de aprofundar e reforçar o conhecimento científico de determinadas áreas e subsidiar a tomada de decisões dos profissionais. 
Para a construção de uma revisão integrativa é necessário seguir seis etapas distintas: a identificação do tema e seleção da hipótese ou questão de pesquisa; estabelecimento de critérios para inclusão e exclusão de estudos/amostragem ou busca na literatura; definição das informações a serem extraídas dos estudos selecionados/ categorização dos estudos; avaliação dos estudos incluídos; interpretação dos resultados; e apresentação da revisão/síntese do conhecimento (Ercole, Melo, \& Alcoforado, 2014).

A abordagem qualitativa exerce um grau de existência que não pode ser avaliado, e que trabalha com fenômenos humanos como as crenças, as aspirações, os valores e as atitudes onde é compreendido como parte da realidade social (Minayo, 2010).

Foi realizada uma busca criteriosa de estudos, para assim serem analisados e discutidos de forma rigorosa, sem plágios e preservando o pensamento dos autores. A coleta de dados foi realizada no mês de março de 2020 nos bancos de dados da Biblioteca Virtual em Saúde (BVS), nas bases de dados SCIELO, LILACS e Google acadêmico, com os seguintes descritores presentes no DECS: Assistência de Enfermagem, Infarto Agudo do Miocárdio, Angioplastia. Como critérios de inclusão foram escolhidos os artigos que apresentavam texto completo, em português e espanhol, no período de 2014 a 2019 e que estavam relacionados ao tema. Foram excluídos: teses, dissertações, artigos pagos, de jornal e textos que não apresentavam relação com a temática e revisões de literatura.

Os dados foram coletados por meio de um formulário que continha as variáveis: Ano de publicação; Abordagem metodológica; Base de dados; Região geográfica; Periódico; Título do artigo; Autor e Contribuições do estudo. Estas variáveis foram dispostas em tabela e quadro, utilizando-se porcentagem simples, e posteriormente, foi realizada a criação de categorias que responderam aos objetivos do estudo, realizando a discussão de acordo com os resultados dos artigos trabalhados.

\section{Resultados e Discussão}

Após seleção realizada na base de dados foram encontrados 402 artigos relacionados aos descritores. Posteriormente a esta etapa, foram selecionados aqueles que se encontravam dentro dos critérios de inclusão propostos, ficando 89 artigos, dos quais foram excluídos 80 , por não se enquadrarem aos respectivos itens.

Depois de realizada a leitura criteriosa dos artigos selecionados, para obtenção daqueles que possuíam relação com a temática, resultou-se em uma amostra final de 9 artigos. 
Research, Society and Development, v. 9, n. 7, e755974878, 2020

(CC BY 4.0) | ISSN 2525-3409 | DOI: http://dx.doi.org/10.33448/rsd-v9i7.4878

A partir do estudo dos artigos estabeleceram-se variáveis relevantes para observação das produções científicas relacionadas à pesquisa, conforme descrito na Tabela 1 .

Tabela 1: Distribuição das produções científicas segundo o Ano de publicação, Abordagem metodológica, Base de dados e Periódico ( $\mathrm{n}=9$ ). Teresina, 2020.

\begin{tabular}{llc}
\hline \multicolumn{1}{c}{ VARIÁVEIS } & N & $\%$ \\
\hline ANO DE PUBLICAÇÃO & 02 & 22.2 \\
2015 & 01 & 11.1 \\
2016 & 03 & 33.3 \\
2017 & 03 & 33.3 \\
2018 & 02 & 22.2 \\
2015 & 01 & 11.1 \\
2016 & & 55,5 \\
ABORDAGEM METODOLÓGICA & 05 & 44,4 \\
Qualitativa & 04 & 11,1 \\
Quantitativa & & 44,4 \\
BASES DE DADOS & 1 & 44,4 \\
LILACS & 4 & 11.1 \\
SCIELO & 4 & 11.1 \\
GOOGLE ACADÊMICO & & 11.1 \\
PERIÓDICO & & 11.1 \\
Caderno de Graduação - Ciências Biológicas e da Saúde UNIT & 01 & 22,2 \\
Ensaios USF & & 11.1 \\
Revista Contexto e saúde & 01 & 11.1 \\
Revista FASEM - Faculdade Serra da Mesa & 01 & 11.1 \\
Revista Científica de Enfermagem & 01 & 02 \\
Revista Brasileira de Enfermagem & 01 & \\
Ciência \& Saúde Coletiva & 01 & \\
Congresso Internacional de Enfermagem & 01 & \\
\hline
\end{tabular}

Fonte: Biblioteca Virtual em Saúde.

Observou-se, conforme a Tabela 1, que os anos de maior número de publicações foram os de 2017 e 2018, ambas com (33.3\%). Em seguida, o ano de 2015 correspondendo a (22.2\%), seguido do ano de 2016 com menor percentual (11.1\%). Os anos de 2014 e 2019 não foram encontrados artigos acerca da temática do estudo. Em todos os outros anos foram encontrados artigos, contribuindo dessa forma, na produção do trabalho acerca da assistência de enfermagem ao paciente com IAM submetido a angioplastia coronariana.

Com relação à abordagem metodológica, a que predominou foi a qualitativa com (55.5\%), seguida da abordagem quantitativa com (44.4\%) das publicações. A cerca das Bases de dados, o Google Acadêmico e Scielo, ambas obtiveram o mesmo percentual com (44.4\%), seguida da Lilacs com menor índice de (11.1\%). 
Research, Society and Development, v. 9, n. 7, e755974878, 2020

(CC BY 4.0) | ISSN 2525-3409 | DOI: http://dx.doi.org/10.33448/rsd-v9i7.4878

O Quadro 1, seguinte, discorre acerca da categorização dos artigos selecionados para desenvolver esse estudo sobre assistência de enfermagem ao paciente com IAM submetido a angioplastia coronariana.

Quadro 1: Agrupamento dos estudos conforme Número do artigo, Título do artigo, Autor, Contribuições do estudo e Categorias.

\begin{tabular}{|c|c|c|c|c|}
\hline $\mathrm{N}^{\circ}$ & Título do artigo & Autor & Contribuições do estudo & $\begin{array}{c}\text { Categor } \\
\text { ias }\end{array}$ \\
\hline 1 & $\begin{array}{l}\text { Aspectos clínico-epidemiológicos de pacientes } \\
\text { submetidos à Intervenção Coronária Percutânea em } \\
\text { hospital universitário. }\end{array}$ & Lima et al & $\begin{array}{l}\text { Aspectos epidemiológicos relacionados aos pacientes } \\
\text { que foram submetidos à intervenção coronária }\end{array}$ & 01 \\
\hline 2 & $\begin{array}{l}\text { Perfil de Pacientes submetidos a cateterismo } \\
\text { cardíaco e Angioplastia em um Hospital Geral }\end{array}$ & Kuhn et al & $\begin{array}{l}\text { Perfil epidemiológico de pacientes que foram } \\
\text { submetidos à intervencão Angioplastia coronariana. }\end{array}$ & 01 \\
\hline 3 & $\begin{array}{l}\text { O perfil das internações e da mortalidade por Infarto } \\
\text { Agudo do Miocárdio no município de XANXERE }\end{array}$ & $\begin{array}{l}\text { Machado, } \\
\text { Hillesheim } \\
\text { \& Ferraz }\end{array}$ & $\begin{array}{l}\text { Perfil da morbimortalidade por Infarto Agudo do } \\
\text { Miocárdio }\end{array}$ & 01 \\
\hline 4 & $\begin{array}{l}\text { Mortalidade por infarto agudo do miocárdio no } \\
\text { brasil e suas regiões geográficas: análise do } \\
\text { efeito da idade-período-coorte }\end{array}$ & Santos et al & $\begin{array}{l}\text { Mortalidade maior em homens quando comparado às } \\
\text { mulheres, em todas as regiões do País por Infarto Agudo } \\
\text { do Miocárdio. }\end{array}$ & 01 \\
\hline 5 & $\begin{array}{c}\text { Cuidados de enfermagem e } \\
\text { paciente submetidos a a anóstico para } \\
\text { coronariana transluminal percutânea }\end{array}$ & Taets & $\begin{array}{l}\text { Cuidados de enfermagem que podem ser aplicados ao } \\
\text { paciente submetido a Angioplastia Coronariana } \\
\text { transluminal Percutânea }\end{array}$ & 02 \\
\hline 6 & $\begin{array}{l}\text { Infarto agudo do miocárdio com supradesnível do } \\
\text { segmento st e a assistência de enfermagem no intra- } \\
\text { hospitalar }\end{array}$ & $\begin{array}{c}\text { Mendes \& } \\
\text { Miranda }\end{array}$ & $\begin{array}{l}\text { Assistência de enfermagem é essencial no tratamento e } \\
\text { recuperação do paciente com IAM. }\end{array}$ & 02 \\
\hline 7 & $\begin{array}{l}\text { Cuidados de Enfermagem no Cateterismo Cardíaco } \\
\text { e Angioplastia Coronariana: Desenvolvimento de } \\
\text { um instrumento. }\end{array}$ & $\begin{array}{l}\text { Régis, Rosa } \\
\text { \& Lunelli }\end{array}$ & $\begin{array}{l}\text { Cuidados de enfermagem prestados ao paciente } \\
\text { submetido ao cateterismo cardíaco e angioplastia } \\
\text { coronariana. }\end{array}$ & 02 \\
\hline 8 & $\begin{array}{l}\text { Assistência de enfermagem a paciente portador de } \\
\text { infarto agudo do miocárdio. }\end{array}$ & \begin{tabular}{|c|} 
Santos, \\
Barbosa, \& \\
Amorim \\
\end{tabular} & $\begin{array}{l}\text { Assistência de enfermagem ao paciente com IAM e } \\
\text { evolução com melhora da estabilidade hemodinâmica. }\end{array}$ & 02 \\
\hline 9 & obre & & & 02 \\
\hline
\end{tabular}

Fonte: Autores.

Após a leitura detalhada dos artigos selecionados observou-se a necessidade de categorizá-los conforme a similaridade dos conteúdos. para isso, foram criadas 02 categorias: categoria 1 - perfil de pacientes com infarto agudo do miocárdio e as implicações para assistência de enfermagem ; categoria 2 - assistência de enfermagem ao paciente submetido a angioplastia coronariana pós infarto agudo do miocárdio.

\subsection{Categoria 1 - Perfil de pacientes com infarto agudo do miocárdio e as implicações para assistência de enfermagem}

Dos 09 artigos analisados, os 04 primeiros enquadraram-se nesta categoria, conforme o exposto no quadro, com abordagem relacionada ao perfil de pacientes acometidos com Infarto Agudo do Miocárdio. O estudo de Lima et al., (2018) abordam sobre uma pesquisa 
realizada em um hospital universitário, onde foram incluídos no estudo 222 pacientes acometidos por IAM, em que prevaleceram no estudo pacientes do sexo masculino $(65,3 \%)$. Da amostra total, 93,6\% tinham idade maior que 50 anos. Diante disso, observa-se que eventos cardiovasculares como o IAM, possuem menor incidência em mulheres, possivelmente pela função protetora do estradiol. Já o estrogênio associa-se a um elevado risco cardiovascular por sua diminuição dos níveis no período pós-menopausa, o que possibilita ao enfermeiro uma maior atenção em sua assistência sobre os fatores quer acometem os indivíduos masculinos.

Em conformidade com os outros autores, Kuhn et al., (2015), aborda em seu estudo que a idade de pacientes acometidos por IAM, encontra-se entre 50 e 70 anos. Em relação ao sexo a predominância foi no sexo masculino, com 59,7\% dos procedimentos realizados. Dessa forma, mostrando-se ser um fator de risco relevante para o entendimento do enfermeiro, contribuindo na assistência prestada as DCV.

Ao analisar as faixas etárias sobre sua contribuição no agravamento do IAM, no estudo de Machado, Hillesheim \& Ferraz (2018) elencaram sobre a mortalidade masculina, que é maior entre os pacientes 50 e 59 anos, sendo que, o risco de mortalidade por IAM em homens é duas vezes mais que o observado em mulheres. Este fator dá sobre mortalidade masculina pode ser contextualizado, em parte, por fatores hormonais protetores femininos e também porque as mulheres são mais assíduas aos serviços de saúde, possibilitando a detecção de problemas de saúde, em tempo de encontrar uma solução para este tipo de agravo.

Em concordância com os autores, Santos et al., (2018) relatam que com o avança da idade, ambos os sexos se predispõe ao aumento progressivo da mortalidade por IAM. Desse modo, evidenciaram quer houve maiores taxas de mortalidade em homens quando comparado às mulheres, em todas as regiões do país.

\subsection{Categorias 2 - Assistência de Enfermagem ao paciente submetido a angioplastia coronariana pós Infarto Agudo do Miocárdio}

Nesta categoria foram enquadrados 05 artigos dos 09 estudos analisados, para retratar a assistência de enfermagem ao paciente submetido à angioplastia coronariana. $\mathrm{O}$ autor Taets (2016), ressalta que quando o enfermeiro depara-se com pacientes acometidos por IAM para realização de Angioplastia Coronariana, é importante um plano de cuidados acerca da assistência, abordando as dificuldades apresentadas por esse procedimento, para obtenção de resultados satisfatórios. Destaca ainda que as complicações vasculares mais importantes 
foram o hematoma no sítio de punção, hematoma retro peritoneal, pseudoaneurisma, fístula arteriovenosa e dissecção arterial e/ou oclusão. Com isso, a equipe de Enfermagem responsabiliza-se pelos cuidados ao cliente, tanto quanto ao conforto, possíveis complicações, e promoção à saúde e bem-estar, descanso e conforto, para corpo e mente.

O estudo dos autores Mendes \& Miranda (2015) abordam que o enfermeiro deve atentar para reações do paciente acometido por IAM, pois o comportamento diferencia-se de paciente para paciente, exigindo do mesmo uma assistência rápida, explicando que é necessário permanecer em repouso absoluto, acalmá-lo, oferecer apoio emocional e falar de maneira calma a fim de tranquilizar o mesmo e assegurar um ambiente sem ruído.

A realização do cuidado pela equipe de enfermagem torna-se relevante com a implantação da Sistematização da Assistência de Enfermagem (SAE), promovendo diagnóstico de Enfermagem, sendo uma das etapas do processo de Enfermagem, obtendo dessa forma, qualificação dos cuidados e das ações prestadas ao paciente. Com um instrumento em mãos, facilita a análise e/ou observação do enfermeiro na realização do cuidado, bem como planejar e registrar suas ações. Desse modo, o enfermeiro deve conhecer os procedimentos realizados, os benefícios, riscos e suas complicações, para, então, conferir intervenções cabíveis e indispensáveis ao seu plano de cuidados (Régis et al., 2017).

O estudo destes autores Régis et al., (2017), ainda destacam que a enfermagem cardiológica, exerce função extremamente importante na realização dos cuidados aos pacientes no pré-procedimento, a atenção durante o procedimento e os cuidados pósprocedimento, não se limitando apenas à realização médica, pelo contrário, é um procedimento realizado em equipe, envolvendo um cuidado multiprofissional de forma holística.

No estudo de Santos et al., (2017) é abordado que a assistência prestada ao paciente em Pós-Angioplastia Coronariana como tratamento do IAM nas primeiras 6 a 12 horas, deve ser capaz de prevenir, identificar e tratar possíveis complicações que possam ser apresentadas pelos pacientes pós-procedimento. O Enfermeiro deverá estimular a autonomia e incentivar no autocuidado, estabelecendo um vínculo terapêutico, o que, quando bem prestado, irá propiciar uma melhor qualidade de vida para o paciente.

$\mathrm{Na}$ assistência de enfermagem em pós-angioplastia coronariana é exigido do Enfermeiro atenção redobrada aos pacientes no fim do procedimento, promover os devidos cuidados e esclarecimento aos familiares, diminuindo assim os níveis de ansiedades dos mesmos, além de gerenciar toda a equipe. Com isso, observa-se que os desafios na assistência de enfermagem ao paciente em pós-angioplastia coronariana estão voltados para a alta 
demanda de cuidados e o gerenciamento da equipe pelo enfermeiro (Santos, Barbosa, \& Amorim).

Corroborando com este estudo, Silva, Silva, \& Fernandes (2017) relatam a importância do aprimoramento constante da assistência do enfermeiro nas complicações cardiovasculares, com base na educação permanente. Desse modo, os serviços de saúde devem proporcionar aos profissionais, ambientes coletivos, possibilitando uma melhor reflexão e análise dos referenciais norteadores do seu saber técnico e cientifico, promovendo uma aprendizagem continua referente às suas necessidades pessoais e profissionais, bem como, um cuidado mais qualificado para seus pacientes.

\section{Considerações Finais}

Esse estudo atendeu aos objetivos propostos e mostrou-se de grande relevância, pois poderá contribuir para o entendimento do enfermeiro na abordagem e na atenção ao paciente com IAM submetido à angioplastia coronariana.

Faz-se necessária a inclusão desse assunto durante o processo de formação desse profissional, no sentido de prepará-lo para uma assistência qualificada, exigindo do mesmo conhecimento específico.

Dessa forma, é relevante destacar que o papel do enfermeiro na assistência ao paciente com IAM submetido à angioplastia coronariana, deve ser enfatizado, especialmente nos cursos de graduação, para que os acadêmicos tenham uma noção de como proceder na vida futura, mediante essas situações.

Treinamento e capacitação contínua de profissionais da enfermagem que lidam com esse tipo de situação para que os mesmos tenham subsídios e forneçam uma assistência eficaz e de qualidade torna-se necessário, para que o paciente tenha um atendimento holístico para sua pronta recuperação.

Com isso, este estudo é pertinente e pode servir de base para realização de outros trabalhos que venham enriquecer a temática, sendo quer, a mesma possui escassez em publicações. Sendo assim, irá proporcionar a outros pesquisadores a buscar por novas atualizações, satisfazendo assim, suas curiosidades em relação a um tema que representa relevantes contribuições. 


\section{Referências}

Barroso TA, Marins LB, Alves R, Gonçalves ACS, Barroso SG \& Rocha GDS. (2017). Associação entre a obesidade central e a incidência de doenças e fatores de risco cardiovascular. International Journal of Cardiovascular Sciences, 30(5), 416-24.

Cunto Taets GG. (2016). Cuidados de enfermagem e diagnósticos para pacientes submetido à angioplastia coronária transluminal percutânea. Revista Recien-Revista Científica de Enfermagem, 6(16), 3-10.

Ercole FF, Melo LSD \& Alcoforado CLGC. (2014). Revisão integrativa versus revisão sistemática. Revista Mineira de Enfermagem, 18(1), 9-12.

Galvão TF \& Pereira MG. (2014). Revisões sistemáticas da literatura: passos para sua elaboração. Epidemiologia e Serviços de Saúde, 23, 183-4.

Kuhn OT, Bueno JFB, Loro MM, Kolankiewicz ACB, Winkelmann ER \& Rosanelli CLP. (2015). Perfil de pacientes submetidos a cateterismo cardíaco e angioplastia em um hospital geral. Revista Contexto \& Saúde, 15(29), 4-14.

Lima MSMD, Dantas RAN, Mendes NPDN, Alves LCDM, Silva TTMD, Brito AGDR \& Dantas DV. (2018). Aspectos clínico-epidemiológicos de pacientes submetidos à Intervenção Coronária Percutânea em hospital universitário. Revista Brasileira de Enfermagem, 71(6), 2883-90.

Machado DP, Cristina A \& Ferraz L. (2018). O perfil das internações e da mortalidade por infarto agudo do miocárdio no município de Xanxerê. Disponível em; http://www.uniedu.sed.sc.gov.br/wp-content/uploads/2016/09/Davi-Provenzi.pdf.

Mendes MM \& Costa Miranda IP. (2015). Infarto Agudo Do Miocárdio Com Supradesnível Do Segmento St E A Assistência De Enfermagem No Intra-Hospitalar. Revista Eletrônica de Ciências Humanas, Saúde e Tecnologia, 4(1), 81-112.

Minayo, M. C. (2002). Pesquisa social: teoria e método. Petrópolis: Vozes. 
Oliveira F, Silva WM \& Fernandes GCG. (2017). Percepção do enfermeiro sobre o atendimento ao paciente com suspeita de infarto agudo do miocárdio. Ensaios USF, 1(1)1-13.

OPAS/OMS Brasil. 2017. OPAS/OMS Brasil -Doenças Cardiovasculares. Available at:< https://www.paho.org/bra/index.php?option=com_content\&view=article\&id=5253:doe ncascardiovasculares\&Itemid=1096> [Accessed 22 May 2020.

Pereira AS, Shitsuka DM, Parreira FJ \& Shitsuka R (2018). Metodologia da pesquisa científica. [e-book]. Santa Maria. Ed. UAB/NTE/UFSM. Disponível em: https://repositorio.ufsm.br/bitstream/handle/1/15824/Lic_Computacao_MetodologiaPesquisa-Cientifica.pdf?sequence $=1$.

Régis AP, Dalla Rosa GC \& Lunelli T. (2017). Cuidados de enfermagem no cateterisno cardíaco e angioplastia coronariana: desenvolvimento de um instrumento. Revista RecienRevista Científica de Enfermagem, 7(21), 3-20.

Santos JD, Meira KC, Camacho AR, Salvador PTCDO, Guimarães RM, Pierin ÂMG, ... \& Freire FHMDA. (2018). Mortalidade por infarto agudo do miocárdio no Brasil e suas regiões geográficas: análise do efeito da idade-período-coorte. Ciênc. \& Saúde Coletiva, 23, 1621-34.

Santos, V. V., Barbosa, V. C. S., \& Amorim, C. F. (2017, December). Assistência de Enfermagem a Paciente Portador de Infarto Agudo do Miocárdio. In Congresso Internacional de Enfermagem. 1(1).

\section{Porcentagem de contribuição de cada autor no manuscrito}

William Da Silva Santos- 40\%

Nyckson Josué De Meneses Claudino Gonçalves- 40\%

Magda Rogéria Pereira Viana- 20\% 